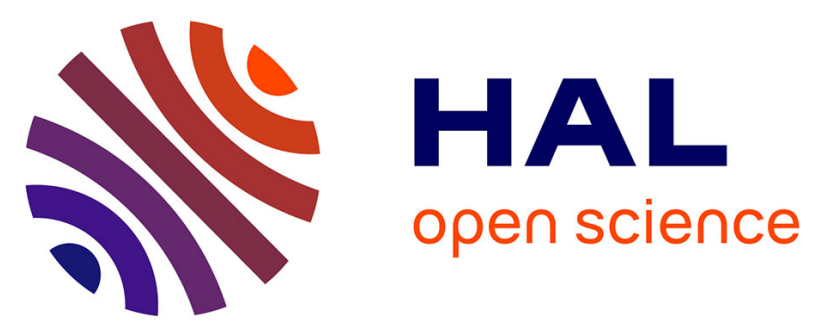

\title{
Torque Prediction Using Stimulus Evoked EMG and its Identification for Different Muscle Fatigue States in SCI Subjects
}

Qin Zhang, Mitsuhiro Hayashibe, Maria Papaiordanidou, Philippe Fraisse, Charles Fattal, David Guiraud

\section{To cite this version:}

Qin Zhang, Mitsuhiro Hayashibe, Maria Papaiordanidou, Philippe Fraisse, Charles Fattal, et al.. Torque Prediction Using Stimulus Evoked EMG and its Identification for Different Muscle Fatigue States in SCI Subjects. EMBC'10: 32nd Annual International Conference of the IEEE Engineering in Medicine and Biology Society "Merging Medical Humanism and Technology", Aug 2010, Buenos Aires, Argentina. pp.3523-3526. lirmm-00502330

\section{HAL Id: lirmm-00502330 \\ https://hal-lirmm.ccsd.cnrs.fr/lirmm-00502330}

Submitted on 13 Jul 2010

HAL is a multi-disciplinary open access archive for the deposit and dissemination of scientific research documents, whether they are published or not. The documents may come from teaching and research institutions in France or abroad, or from public or private research centers.
L'archive ouverte pluridisciplinaire HAL, est destinée au dépôt et à la diffusion de documents scientifiques de niveau recherche, publiés ou non, émanant des établissements d'enseignement et de recherche français ou étrangers, des laboratoires publics ou privés. 


\title{
Torque Prediction Using Stimulus Evoked EMG and its Identification for Different Muscle Fatigue States in SCI Subjects
}

\author{
Qin Zhang, Mitsuhiro Hayashibe, Maria Papaiordanidou, Philippe Fraisse, Charles Fattal and David Guiraud
}

\begin{abstract}
Muscle fatigue is an unavoidable problem when electrical stimulation is applied to paralyzed muscles. The detection and compensation of muscle fatigue is essential to avoid movement failure and achieve desired trajectory. This work aims to predict ankle plantar-flexion torque using stimulus evoked EMG (eEMG) during different muscle fatigue states. Five spinal cord injured patients were recruited for this study. An intermittent fatigue protocol was delivered to triceps surae muscle to induce muscle fatigue. A hammerstein model was used to capture the muscle contraction dynamics to represent eEMG-torque relationship. The prediction of ankle torque was based on measured eEMG and past measured or past predicted torque. The latter approach makes it possible to use eEMG as a synthetic force sensor when force measurement is not available in daily use. Some previous researches suggested to use eEMG information directly to detect and predict muscle force during fatigue assuming a fixed relationship between eEMG and generated force. However, we found that the prediction became less precise with the increase of muscle fatigue when fixed parameter model was used. Therefore, we carried out the torque prediction with an adaptive parameters using the latest measurement. The prediction of adapted model was improved with $16.7 \%-50.8 \%$ comparing to the fixed model.
\end{abstract}

\section{INTRODUCTION}

Muscle fatigue is one of the major factors which limit the application of functional electrical stimulation (FES). It is essential for spinal cord injured (SCI) patients to monitor and compensate muscle fatigue due to the lack of sensory feedback to indicate fatigue. In the previous researches, one reported solution is to use stimulus evoked EMG (eEMG) signal for a prediction of the varying muscle force. It is important to predict generated force when FES is applied along with muscle fatigue.

Many studies have investigated the eEMG and torque manifestations of muscle fatigue induced by FES during static and dynamic contractions. Under continuous stimulation, an exponential curve was utilized to express this relationship on SCI patients [1] [2]. The generated torque induced by sustained stimulation represented three states: potentiation, fatigue and maximal fatigue. A high correlation but different correlation between the generated torque and eEMG was found in these three states [3]. In [4], the relationship was characterized by Hammerstein model, where the mean absolute value (MAV) of eEMG was the input and the knee

The first author is partly supported by China Scholarship Council.

Q. Zhang, M. Hayashibe, P. Fraisse, and D. Guiraud are with INRIA Sophia-Antipolis, DEMAR project, LIRMM, France. qzhang, hayashibe, fraisse, guiraud @lirmm.fr

M. Papaiordanidou is with University of Montpellier I, Motor Efficiency and Deficiency Laboratory, Montpellier, France.

C. Fattal is with Propara Rehabilitation Center, Laboratory of Clinical Neurophysiology, Montpellier, France. torque was output. They considered a fixed parameters model which was identified during a previous day's test session could predict the majority of the potentiation and fatigue response when muscle was fatigued.

Furthermore, a lot of factors can affect the eEMG-force relationship, such as the stimulation pattern (variable versus constant stimulation, high frequency versus low frequency stimulation), the muscle property (fast versus slow muscle) and muscle contraction type (dynamic versus static contraction). Moreover, the muscle fatigue is often a combination of three types of fatigue phenomena (excitation, contractile and ischemic fatigue) which also makes the relationship more complicated. We also need to consider muscle recovery process if there are some intervals between stimulations. Therefore, it is difficult to precisely predict generated force when eEMG-force relationship has variation due to these factors. In our work, we aimed to find a solution to predict torque during different muscle fatigue level induced by intermittent stimulation in paralyzed muscles.

The fatigue process was compared between continuous and intermittent stimulation [5]. They found different manifestations of evoked torque and eEMG with the same stimulation intensity during stimulation. EEMG-force relationship was indicated depending on the fatigue state of the muscle [6]. Both peak-to-peak amplitude and force behaved quite differently during recovery which was represented by different recovery velocity after rest. A metabolite-based fatigue function was proposed in prediction of the force history of a paralyzed quadriceps [7]. Disassociation during recovery process between torque and eEMG was found in [8]. They also suggested an adaptive tuning of the parameters of eEMG in predicting the stimulated force. However, in their work, a feasible prediction method for varying force was not proposed and implemented yet.

The aim of this study is to propose a feasible solution to predict electrically stimulated force by adjusting the eEMGforce model parameters. Through the fatigue experiments on five SCI patients, we verified our approach and confirmed that the prediction could be improved with adaptive model parameters for different muscle fatigue states for all subjects.

\section{METHODS}

\section{A. Subjects and Experimental Setup}

Five SCI subjects were recruited for this experiment (see TABLE I). The study was approved by the ethical committee and all subjects signed informed consent forms. During each experiment, triceps surae muscle group was stimulated by surface electrodes $(10 \mathrm{~cm} \times 3 \mathrm{~cm})$. One electrode was placed 
$5 \mathrm{~cm}$ beneath the popliteal cavity and the other beneath the insertion point of the medial and lateral gastrocnemius on the Achilles tendon. The muscle group was stimulated with amplitude modulation at a constant frequency $(30 \mathrm{~Hz})$ and constant pulse-width $(450 \mu \mathrm{s})$, under isometric conditions by Cefar physio4 stimulator.

TABLE I

PATIENT CONFIGURATIONS

\begin{tabular}{|c|c|c|c|c|c|}
\hline $\begin{array}{c}\text { Test } \\
\text { Subject }\end{array}$ & $\begin{array}{c}\text { Age } \\
\text { (years) }\end{array}$ & $\begin{array}{c}\text { Weight } \\
(\mathrm{kg})\end{array}$ & $\begin{array}{c}\text { Height } \\
(\mathrm{cm})\end{array}$ & $\begin{array}{c}\text { Level } \\
\text { of injury }\end{array}$ & $\begin{array}{c}\text { Months } \\
\text { post injury }\end{array}$ \\
\hline S1 & 39 & 50 & 169 & T6 & 3 \\
\hline S2 & 22 & 54 & 172 & C7 & 30 \\
\hline S3 & 26 & 64 & 192 & T6 & 36 \\
\hline S4 & 32 & 61.5 & 177 & C5 & 8 \\
\hline S5 & 48 & 76 & 177 & T6 & 18 \\
\hline
\end{tabular}

The subjects were seated on the chair with their ankle at $90^{\circ}$, while the foot was strapped on the pedal. Isometric ankle plantar-flexion torque was measured using a calibrated dynamometer (Biodex), interfaced with an acquisition system (Biopac MP100). Evoked EMG activity of gastrocnemius and soleus was respectively recorded using two bipolar $\mathrm{AgCl}$ surface electrodes positioned over each muscle belly along the muscle fiber direction. The skin was shaved to minimize the impedance. The interelectrode spacing in each pair of EMG electrodes was $20 \mathrm{~mm}$. The reference electrode was placed on the patella of another leg. The EMG signal was amplified (gain 1,000) and sampled at $4 \mathrm{KHz}$ along with the ankle torque.

\section{B. Experimental Protocols}

In all the experiments, the right triceps surae muscle was stimulated. The experiment of each subject consists of three sessions: fatigue-inducing test, fatigue-recovery test and random test, as shown in Fig. 1. In order to induce muscle fatigue, three series of five trains (1s ramp, $2 \mathrm{~s}$ holding, $1 \mathrm{~s}$ falling, $2 \mathrm{~s}$ interval, $30 \mathrm{~Hz}, 450 \mu \mathrm{s}$, at intensity evoking $50 \%$ of maximum torque) were applied for subject S1 and S2. Four series of the same stimulation trains were applied to S3. Five series were applied to S4 and S5. The stimulation duration in one fatigue test series is $30 \mathrm{~s}$. In fatigue-recovery test, one train at intensity where maximal torque was obtained was delivered to the muscle just before or after each fatigueinducing test session. After the last post fatigue stimulation (postE), the same stimulation was applied every 5 minutes for 15 minutes. In random test, the stimulation amplitude was increased from zero to a randomly determined value, and then symmetrically decreased, totally for 2 minutes.

\section{Data Analysis}

Under muscle stimulation, the muscle response (Mwave) is normally contaminated by stimulation artifacts. We removed the stimulation artifacts by means of blanking window. A $3 \mathrm{~ms}$ time window was selected at the onset of the stimulation to suppress the artifacts. Measured data of ankle torque was lowpass filtered (6th order, cutoff frequency $100 \mathrm{~Hz}$ ) and then

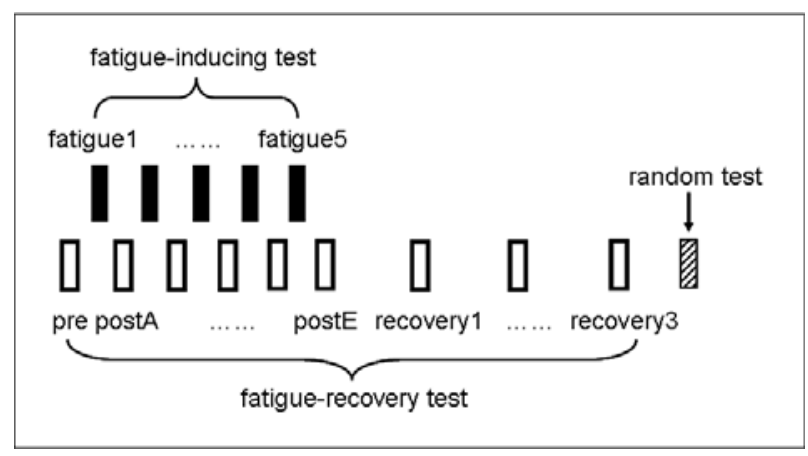

Fig. 1. Schematic representation of the experimental session.

offset with respect to the baseline of the torque measurement without stimulation. The measured eEMG data was lowpass filtered (6th order, cutoff frequency $300 \mathrm{~Hz}$ ) and then divided into epochs with each epoch containing one Mwave. For predicting the ankle torque, the MAV was calculated every 5 epochs. The corresponding mean torque was simultaneously calculated during the same time window. In this work, eEMG of soleus was used as representative muscle response of triceps surae muscle group to make correlation with ankle plantar flexion torque.

The torque measurement and the eEMG measurement were used to identify the parameters of mathematical models for muscle torque prediction. A Hammerstein model was used to represent the contraction dynamics as proposed in [4]. The main function of this model can be written as follows:

$$
y(t)=\sum_{i=1}^{l} a_{i}[y(t-i)]+\sum_{j=1}^{m} \sum_{k=1}^{n} b_{j k}[x(t-j)]^{k}+c_{0}
$$

where $y(t)$ is the torque output at $t$ instant, $x(t)$ is the input, in this study, we use MAV of evoked EMG as input. Term $c_{0}$ is used to fit any offset of the output torque. We chose $n=3$ as in [4]. We assumed different model order $(l, m)$ to identify and validate the model. We found that the prediction error was minimized by model order $(l=3, m=$ $4)$. Recursive least square method was used to identify the parameters $\left(a_{i}, b_{j k}\right)$. In this model, the past torque $y(t-i)$ has two possibilities: i) past measured torque and ii) past predicted torque. In our work, we applied the former method for model identification and prediction, the latter one only for torque prediction. In the second approach, the model output $\widehat{y}_{p}(t)$ based on the identified parameters can be computed as follows:

$$
\widehat{y}_{p}(t)=\begin{aligned}
& f[x(t-1), x(t-2), \ldots x(t-m), \\
& \left.\widehat{y}_{p}(t-1), \widehat{y}_{p}(t-2), \ldots \widehat{y}_{p}(t-l)\right]
\end{aligned}
$$

When force measurement is not available, the second approach makes it possible to use eEMG as a synthetic force sensor. In this case, we initialized the predicted torque at zero when no stimulation was delivered to the muscle. 


\section{RESULTS}

\section{A. Muscle Fatigue and Recovery Process}

In every fatigue-recovery test, the plateau that torque arrived at the maximal value was selected to observe the relationship between torque and eEMG. The mean torque and MAV were calculated over the selected duration. The standard deviations were obtained simultaneously. As depicted in Fig. 2(a), the torque output of ankle plantar flexion gradually declined after each fatigue-inducing session. After all the fatigue inducing test sessions, the torque declined to around $90 \%$ of initial torque in all subjects. After 5 minutes rest, the mean torque recovered less than $5 \%$ and then maintained at the same level in all subjects. For subject S1, the mean torque delayed to recover with 10 minutes rest. However, torque transition in fatigue generally showed similar tendency for all subjects. The reason of small recovery can be considered due to intermittent stimulation. Long term stimulation in intermittent way produced real maximal fatigue state. The MAV of eEMG represented different transition among these subjects as depicted in Fig. 2(b). Even the same tendency can be found in subject S3 and S5, different tendency was found among these 5 subjects. The result of S3, S5 showed simple decrease of eEMG due to fatigue, $\mathrm{S} 4$ showed potentiation of eEMG, S1, S2 represented somewhat combined characteristics of fatigue and potentiation. Here, we do not focus on the understanding of the different characteristics. However, we could confirm that eEMG-torque relationship was gradually varying in this intermittent fatigue protocol.
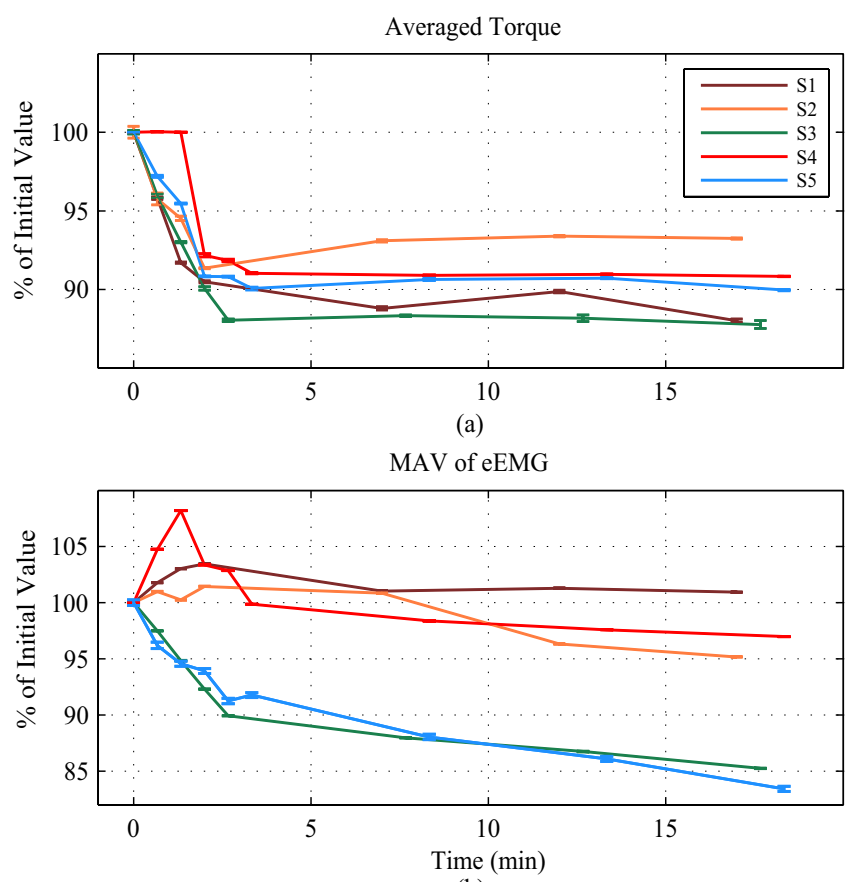

(b)

Fig. 2. (a) Averaged torque and (b) MAV of eEMG during the fatiguerecovery processes. The results were represented as means \pm standard deviation.

\section{B. Model Identification and Validation}

The two approaches of model prediction we explained in section II.C were carried out using random test data. Fig. 3 is the prediction result on subject S5. During 97s random stimulation, the data of the first $68 \mathrm{~s}$ was used for model identification. And then the prediction was implemented for the same data based on measured eEMG and past measured torque to verify the validity of identification. The prediction for the data after 68s was based on measured eEMG and past predicted torque. The corresponding prediction errors (MSE) are shown in the figure.

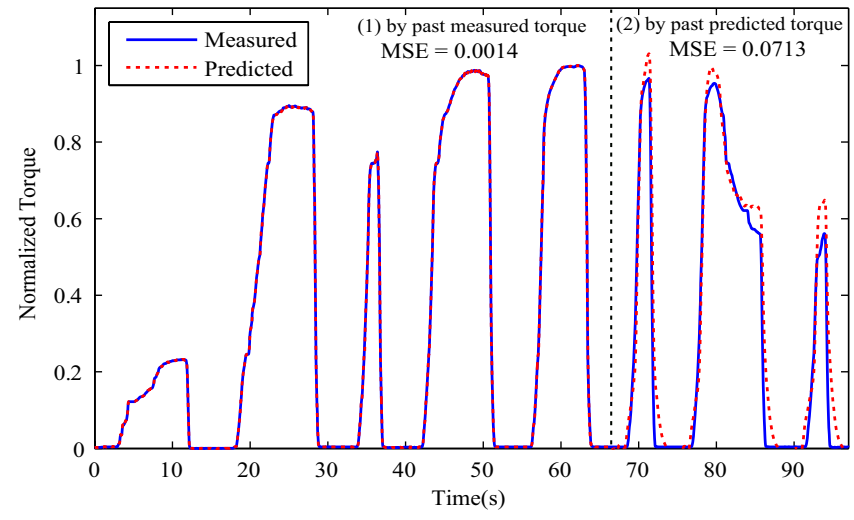

Fig. 3. The measured and predicted torque obtained by eEMG-torque model. The marker line is shown to separate the data into two parts. (1) The data was used for model identification through recursive least square method. The prediction was based on the measured eEMG and past measured torque for these data. (2) The prediction was based on the measured eEMG and past predicted torque for these data.

In this random stimulation test, we could confirm the feasibility of model identification and its prediction performance. For the next step, we need to investigate it in different muscle fatigue states to verify model predictability in fatiguing condition.

We tried the torque prediction using the fatigue-inducing data sets of 5 SCI patients. The first trial of fatigue-inducing test was used to identify the model parameters. The identified model was then cross-validated for all the rest trials. We name this process prediction with fixed parameters model. The prediction errors are shown in TABLE II. We found that with fixed model parameters, the prediction error became higher with time increasing. Thus, we assumed that if we identified the model again with the data in previous trial, the prediction could be improved. Then, we predicted the torque based on the adapted model parameters which were obtained using the previous trial. For example, we predict the torque of fatigue 3 based on the model parameters obtained with fatigue2. We call this process prediction with adapted parameters model. The prediction errors with adapted eEMGtorque model for all subjects are shown in TABLE II. For example, in fatigue3 of subject S3, the MSE with adapted model is 0.0345 by contrast with 0.0639 with the fixed model. The prediction of adapted model was improved with $16.7 \%-50.8 \%$ comparing to the fixed model in all subjects. 
In Fig. 4, the predictions with different approaches (fixed and adapted model) and in different fatigue condition on subject S3 are shown. Due to small difference between fatigue 3 and fatigue 4 , fatigue 3 is not plotted in this figure. The blue solid line respectively indicates the measured torque of fatigue1, fatigue 2 and fatigue 4 in fatigue-inducing test. The red dotted line denotes the corresponding predicted torque based on fixed model parameters which were obtained from fatigue1. The black dashdotted line represents the predicted torque of fatigue 4 based on adapted model parameters which were identified using the previous trial (fatigue3). All the torques were normalized by the maximal measured torque in fatigue 1 . We found that the torque declined with the same stimulation as a result of muscle fatigue. The fixed model could be still used for the torque prediction. However, in comparison with fatigue 1 and fatigue2, the prediction of fatigue4 became less precise based on fixed model. Whereas the adapted model contributed to improve the torque prediction accuracy as the black dashdotted line shown.

The same attempt was also applied to random test data. We found that during 2 minutes stimulation, the eEMGtorque relationship varied after $80 \mathrm{~s}$. When adapted model parameters were used, the prediction was also improved to correspond to the change in eEMG-torque relationship.

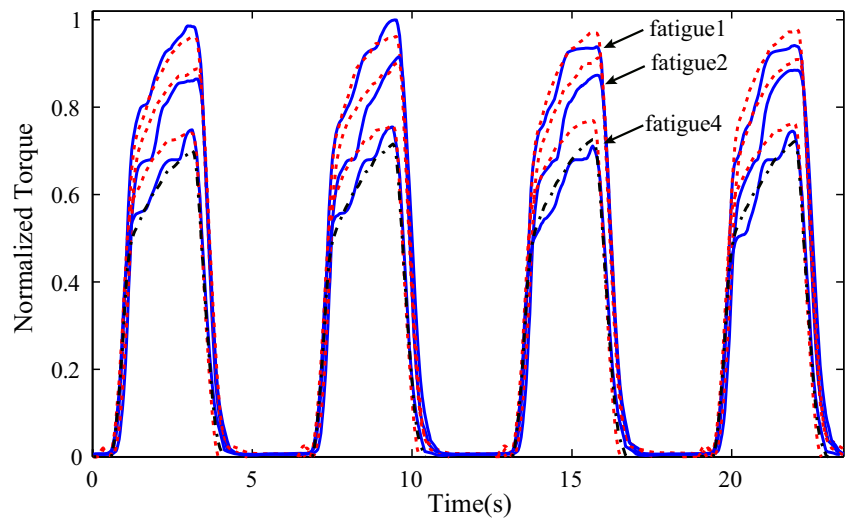

Fig. 4. The measured and predicted torque obtained by eEMG-torque model in fatigue-inducing protocol. The blue solid line indicates the measured torque. The red dotted line represents the corresponding predicted torque based on fixed model. The black dashdotted line represents the torque prediction of fatigue 4 based on adapted model. All the torques are normalized with the maximal measured torque of fatigue1.

\section{CONCLUSIONS AND FUTURE WORKS}

The present work investigated the torque prediction during electrically induced muscle fatigue using myoelectric activities for paralyzed muscles. In previous studies, some researchers considered stimulus evoked EMG could be used directly for monitoring muscle fatigue or predicting generated force. However, other researchers found the relationship between eEMG and force could be changed by recovery or some other influences [8]. We found that a fixed parameters model as in [4] can still predict approximate force decreased by muscle fatigue. However, as reported in other
TABLE II

SUMMARY OF THE PREDICTION ERROR OBTAINED WITH FIXED OR ADAPTED EEMG-TORQUE MODEL

\begin{tabular}{|c|c|c|c|c|c|c|}
\hline \multirow{2}{*}{ Subject } & \multirow{2}{*}{ Model } & \multicolumn{5}{|c|}{ Mean Square Error } \\
\cline { 3 - 7 } & & F1 & F2 & F3 & F4 & F5 \\
\hline \multirow{2}{*}{ S1 } & Fixed & 0.0381 & 0.1009 & 0.2037 & $/$ & $/$ \\
\cline { 2 - 7 } & Adapted & $/$ & $/$ & 0.1272 & $/$ & $/$ \\
\hline \multirow{2}{*}{ S2 } & Fixed & 0.0642 & 0.0767 & 0.1559 & $/$ & $/$ \\
\cline { 2 - 7 } & Adapted & $/$ & $/$ & 0.1298 & $/$ & $/$ \\
\hline \multirow{2}{*}{ S3 } & Fixed & 0.0253 & 0.0467 & 0.0639 & 0.0572 & $/$ \\
\cline { 2 - 7 } & Adapted & $/$ & $/$ & 0.0345 & 0.0254 & $/$ \\
\hline \multirow{2}{*}{ S4 } & Fixed & 0.0511 & 0.0520 & 0.0575 & 0.0702 & 0.0631 \\
\cline { 2 - 7 } & Adapted & $/$ & $/$ & 0.0480 & 0.0447 & 0.0438 \\
\hline \multirow{2}{*}{ S5 } & Fixed & 0.0594 & 0.0641 & 0.0591 & 0.0597 & 0.0715 \\
\cline { 2 - 7 } & Adapted & $/$ & $/$ & 0.0457 & 0.0447 & 0.0476 \\
\hline
\end{tabular}

* F1-F5 respectively denotes the trial fatigue1-fatigue5 of fatigue-inducing test session.

literatures, eEMG-torque relationship varied especially in the long term intermittent fatigue protocol. We found that an adaptively modified model could improve the prediction ability for all subjects. The trend of eEMG-torque differently changed for different subjects and it is difficult to explain the mechanism. However this relationship in one subject gradually changed with muscle fatigue. Therefore, the effect of adaptive identification should be significant and it can be said that this approach can contribute to FES control with fatigue consideration. Future work will be to extend the present approach to on-line automatic identification and adaptive control for FES system.

\section{ACKNOWLEDGMENTS}

Thanks for Patrick Benoit for his therapeutic assistance of patients during the experiments.

\section{REFERENCES}

[1] J. Mizrahi, M. levy, H. Ring, E. Isakov, and A. Liberson, EMG as an indicator of fatigue in isometrically FES-activated paralyzed muscles, IEEE Trans. on Neural Systems and Rehabilitation, vol. 2, 1994, pp $57-65$.

[2] D. Tepavac and L. Schwirtlich, Detection and Prediction of FESInduced Fatigue, J. Electromyogr. Kinesiol., vol. 7, 1997, pp 39-50.

[3] A. Erfanian, H. J. Chizeck and R. M. Hashemi, "ExcitationContraction Fatigue During Sustained Electrical Stimulation of Paralyzed Muscle", in 18th Annual International Conference of the IEEE Engineering in Medicine and Biology Society, Amsterdam, 1996, pp 1460-1461.

[4] A. Erfanian, H. J. Chizeck, and R. M. Hashemi, Using Evoked EMG as a Synthetic Force Sensor of Isometric Electrically Stimulated Muscle, IEEE Trans. on Biomedical Engineering, vol.45, 1998, pp 188-202.

[5] J. J. Chen and N. Y. Yu, The Validity of Stimulus-Evoked EMG for Studying Muscle Fatigue Characteristics of Paraplegic Subjects During Dynamic Cycling Movement, IEEE Trans. on Rehabilitation engineering, vol. 5, 1997, pp 170-178.

[6] J. Mizrahi, E. Isakov and Z. Suzak, Myoelectric and Force Characteristics in Transcutanteous Isometric FES, Basic Appl. Myol., vol. 4, 1994, pp 147-154.

[7] O. Levin and J. Mizrahi, EMG and metabolite-based prediction of force in paralyzed quadriceps muscle under interrupted stimulation, IEEE Trans. on Neural Systems and Rehabilitation, vol. 7, 1999, pp 301-314.

[8] N. Y. Yu and S. H. Chang, "Mechanical and Electromyographic Response to Stimulated Contractions in Paralyzed Tibialis Anterior Post Fatiguing Stimulations", in 13th International Conference on Biomedical Engineering, Singapore, 2009, pp 1667-1671. 